Bull. Korean Math. Soc. 51 (2014), No. 2, pp. 457-478

http://dx.doi.org/10.4134/BKMS.2014.51.2.457

\title{
HOPF BIFURCATION OF CODIMENSION ONE AND DYNAMICAL SIMULATION FOR A 3D AUTONOMOUS CHAOTIC SYSTEM
}

\author{
Xianyi Li AND ZhengXin Zhou
}

\begin{abstract}
In this paper, a 3D autonomous system, which has only stable or non-hyperbolic equilibria but still generates chaos, is presented. This system is topologically non-equivalent to the original Lorenz system and all Lorenz-type systems. This motivates us to further study some of its dynamical behaviors, such as the local stability of equilibrium points, the Lyapunov exponent, the dissipativity, the chaotic waveform in time domain, the continuous frequency spectrum, the Poincaré map and the forming mechanism for compound structure of its special cases. Especially, with the help of the Project Method, its Hopf bifurcation of codimension one is in detailed formulated. Numerical simulation results not only examine the corresponding theoretical analytical results, but also show that this system possesses abundant and complex dynamical properties not solved theoretically, which need further attention.
\end{abstract}

\section{Introduction}

Since the first eminent chaotic attractor was in 1963 found in a simple mathematical model of a weather system which consists of three link nonlinear differential equations, i.e., the Lorenz system [18, 28], the study of chaotic behaviors in nonlinear systems has attracted great attention due to many of possible applications in various fields of science and technology, including fluid mixing [23], secure communication [1], heating [29], and some other scientific and engineering applications [2]. In addition, desirable research interests have been made in not only searching for new chaotic attractors (such as the Chen system [8], the Lü and Chen system [19], the Lorenz system family [3], the conjugate Lorenz-type system [16], [37], etc.) or studying chaos control and chaos synchronization [10], but also theoretically analyzing the local and global characteristics which are essential for the better understanding of what is meant

Received October 22, 2012; Revised August 2, 2013.

2010 Mathematics Subject Classification. 93D05, 65P20, 65P30.

Key words and phrases. 3D autonomous system, chaos, Hopf bifurcation of codimension one, project method, Lyapunov exponent.

This work is partly supported by NNSF of China (grant: 10771094), the NSF of Jiangsu Province (grant: BK2012682) and the NSF of Yangzhou University. 
by chaos. In particular, concerning the Chen system $[9,13,14,15,21]$, the Lü system [38, 39], and others [11], there have been some detailed bifurcation analysis like pitchfork bifurcation, Hopf bifurcation, homoclinic bifurcation, tangent bifurcation, and some other complex dynamical behaviors, which all show that those systems have rich nonlinear dynamics and are of significance in practical applications.

It should be noted that one commonly used analytic criterion for generating and proving chaos in autonomous systems is based on S̆ilnikov's fundamental work [26, 27] and its subsequent embellishment and slight extension [25]. All the excellent results require such systems to have at least an unstable equilibrium. Indeed, in 1976, Rössler conducted important work that rekindled the interest in low-dimensional dissipative dynamical systems [24], then Sprott embarked upon an extensive search for autonomous 3D chaotic systems with fewer than seven terms in the right-hand side of such models. He considered general 3D ordinary differential equations with quadratic nonlinearities and found by computer simulation 19 simple 3D quadratic autonomous chaotic systems with none, one equilibrium or two equilibria [29, 30, 31].

Among these chaotic systems, an interesting phenomenon has been found that some 3D autonomous chaotic systems have three particular fixed points:

1) one saddle and two unstable saddle-foci, for example, in the Lorenz system [18], the Chen system [8], the Lü system [19], and the conjugated Lorenz-type system [37];

2) one saddle and two stable fixed points, such as in [36];

3) two unstable saddle-foci, such as in the original Rössler system [24] and the diffusionless Lorenz system [33].

These systems, together with some of other known systems, basically demonstrate the point of view that those systems generating chaos have at least an unstable equilibrium point.

Therefore, researchers can not help asking a question: whether or not are there 3D autonomous chaotic systems only with stable equilibria and at most six term including nonlinearities such that the topological structures of such chaotic systems are different? So far, Wei and Yang [35] have found such a 3D chaotic system with six terms including two nonlinear terms, one of which has the form of exponential function, namely, the following 3D autonomous system:

$$
\left\{\begin{array}{l}
\dot{x}=a(y-x), \\
\dot{y}=-b y+m x z, \\
\dot{z}=n-e^{x y},
\end{array}\right.
$$

where $a, b, m$ and $n$ are real numbers.

Whether or not are there other simpler 3D autonomous chaotic systems with such properties? Motivated by the former work and this question, in this paper 
we introduce the following $3 \mathrm{D}$ chaotic system with such properties:

$$
\left\{\begin{array}{l}
\dot{x}=a(y-x) \\
\dot{y}=-x z \\
\dot{z}=-b+x y+e x^{2}
\end{array}\right.
$$

where $a, b>0$ and $e>-1$, are real numbers.

Obviously, the nonlinear terms of this system are not of exponential type [35] but polynomial type with three quadratic terms. It will be demonstrated in the sequel that this system may generate a double-scroll chaotic attractor under the different parameters which show the different types of equilibria:

1) all of its equilibria are unstable (trivial case);

2) all of its equilibria are non-hyperbolic (new case);

3) all of its equilibria are stable (nontrivial case).

The terms in the right hands of the system (1.1) are the polynomials with the orders not large than two. Relatively speaking, the system (1.1) is simpler than the system [35] with exponential function as nonlinear term.

Compared with the known Lorenz-type system, it is easy to see that the system (1.1) is not a special case of the unified Lorenz-type system [37] and possess different topological structure. Indeed, according to the work of Čelikovský and Chen $[3,4,5]$, the generalized Lorenz system has the following form:

$$
\dot{x}=\left(\begin{array}{cc}
B & 0 \\
0 & \lambda_{3}
\end{array}\right) x+x_{1}\left(\begin{array}{ccc}
0 & 0 & 0 \\
0 & 0 & -1 \\
0 & 1 & 0
\end{array}\right) x
$$

where $x=\left(x_{1}, x_{2}, x_{3}\right)^{T}, \lambda_{3} \in \mathbb{R}$, and $B$ is a $2 \times 2$ real matrix

$$
B=\left(\begin{array}{ll}
b_{11} & b_{12} \\
b_{21} & b_{22}
\end{array}\right)
$$

with the eigenvalues $\lambda_{1}, \lambda_{2} \in \mathbb{R}$, satisfying $\lambda_{1}>0$ and $\lambda_{2,3}<0$. If one denotes the generalized Lorenz system by the sum of the linear part and the nonlinear part, i.e., $\dot{x}=A x+F(x)$, where $A=\left(a_{i j}\right)_{3 \times 3}$, then, for the coefficient matrix $A$, the Lorenz system satisfies the condition $a_{12} a_{21}>0$ while the Chen system satisfies the condition $a_{12} a_{21}<0$ (So, the Chen system is called a dual system of Lorenz system in the sense defined by Čelikovský and Vaěček $[6,7])$, and the Lü system [32] satisfies the condition $a_{12} a_{21}=0$, representing the transition between the Lorenz system and the Chen system.

The system (1.1), however, correspondingly takes this form

$$
\dot{x}=\left(\begin{array}{cc}
B & 0 \\
0 & \lambda_{3}
\end{array}\right) x+x_{1}\left(\begin{array}{ccc}
0 & 0 & 0 \\
0 & 0 & -1 \\
e & 1 & 0
\end{array}\right) x+\left(\begin{array}{c}
0 \\
0 \\
-b
\end{array}\right)
$$


which contains the chaotic system proposed by Munmuangsaen and Srisuchinwong [22] when $a=5, b=90, e=0$ (At this time

$$
B=\left(\begin{array}{ll}
-5 & 5 \\
0 & 0
\end{array}\right)
$$

and the Hopf bifurcation does not occur in the special system). It is immediately clear that the system (1.1) is not topologically equivalent to the original Lorenz system or the other Lorenz-like systems. We find that, anyway, when

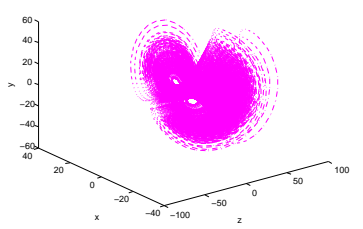

(a) Chaotic attractor of the system (1.1)

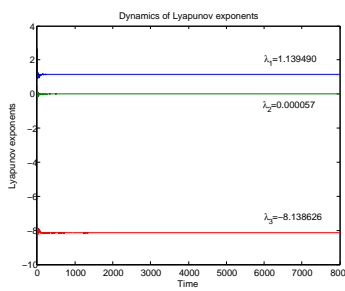

(b) Lyapunov exponents of the system (1.1)

FIG. 1.1 Chaotic attractor and Lyapunov exponents of system $(1.1)$ with $(a, b, e)=(7,50,1)$ and the initial condition $\left(x_{0}, y_{0}, z_{0}\right)=(1,1,1)$.

$(a, b, e)=(7,50,1)$, the system (1.1) has the following Lyapunov exponents: $L_{1}=1.139490, L_{2}=0.000057$ and $L_{3}=-8.138626$. This means that the system (1.1) would produces chaotic phenomenon. Numerical simulation results demonstrate that the system (1.1) indeed possesses strange attractors. To see this, refer to Fig.1.1.

Remark 1.1. The above Lyapunov exponents are obtained under the condition that one uses Matlab with the recurrent number about 8000 . If the recurrent number is less than 2500 , then $L_{2} \approx 0.000166$. Even if so, one should regard the sign of $\left(L_{1}, L_{2}, L_{3}\right)$ as $(+, 0,-)$, not $(+,+,-)$, because "+" and "-" are respectively related to the expansion and contraction direction and determine the expansion and contraction of the attractor in the phase space while " 0 " is related to the critical nature between the expansion and contraction of the attractor in the phase space. In fact, $\left|L_{2}\right|<<\min \left\{\left|L_{1}\right|,\left|L_{3}\right|\right\}$ at this time. Note that $L_{1}, L_{2}$ and $L_{3}$ are denoted as $\lambda_{1}, \lambda_{2}$ and $\lambda_{3}$ in the Figs 1.1(b), 3.1(b), 3.2(b) and $3.3(\mathrm{~b})$.

By comparing the structure of these chaotic attractors in Fig. 1.2, one can find that the structure of the chaotic attractor in the system (1.1) is completely different from that in the Lorenz system [28] or the Chen system [8] or the Lü and Chen system [19], the Yang and Chen system [36] etc. This discovery motivates us to further study what kind of new dynamics this system possesses. 


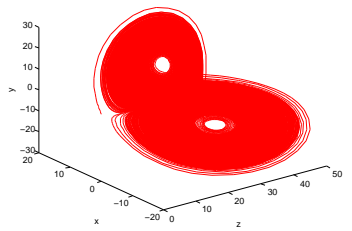

(a) Chaotic attractor of the Lorenz system $\dot{x}=a(y-x), \dot{y}=$ $c x-y-x z, \dot{z}=-b z+x y$ with $(a, b, c)=(10,8 / 3,28)$.

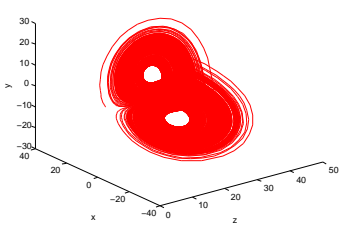

(c) Chaotic attractor of the Lü and Chen system $\dot{x}=a(y-$ $x), \dot{y}=c y-x z, \dot{z}=-b z+x y$ with $(a, b, c)=(36,3,20)$.

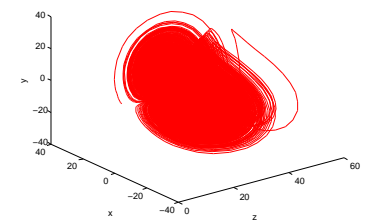

(b) Chaotic attractor of the Chen system $\dot{x}=a(y-x), \dot{y}=(c-$ a) $x+c y-x z, \dot{z}=-b z+x y$ with $(a, b, c)=(35,3,28)$.

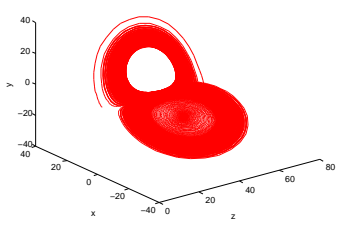

(d) Chaotic attractor of the YC system $\dot{x}=a(y-x), \dot{y}=c x-$ $x z, \dot{z}=-b z+x y$ with $(a, b, c)=$ $(35,3,35)$.

FIG. 1.2 The chaotic attractor of all above known Lorenztype system with the same initial condition $\left(x_{0}, y_{0}, z_{0}\right)=$ $(1,2,3)$.

Generally speaking, it is relatively easy to understand that the instability of equilibrium (equilibria) in a system may lead to the occurrence of chaos. However, the system (1.1) still produces chaos although its two equilibria are stable $^{1}$. Therefore, some physical essences for chaos need people's thorough understanding.

The fact that a system with only stable equilibrium point (points) still may generate chaos maybe provides some new insights into the forming mechanics of chaos. This is also our main purpose to present this paper. Aiming to contribute to the better understanding for the dynamics of this new chaotic system, we present some new results that the system (1.1) can produce chaotic attractor coexistence with three types of equilibria: saddle-foci, non-hyperbolic and stable.

The rest of this paper is organized as follows. In Section 2, the local stability and bifurcation of the system are presented, especially for the computation of Hopf bifurcation of codimension one. Some other dynamical properties, such as the Lyapunov exponent, the dissipativity, the chaotic waveform in

\footnotetext{
${ }^{1}$ It is known recently that Professor Guanrong Chen and his coauthor found that a 3D autonomous system with a unique stable equilibrium point also generates chaos.
} 
time domain, the continuous frequency spectrum, the Poincaré map and the forming mechanism for compound structure of its special cases, the coexistence of chaotic attractor with three type of equilibria: saddle-foci, non-hyperbolic and stable, and the sensitivity condition for the dynamics of the system with fixed parameters values, are formulated in Section 3. The forming mechanism of its compound structures are also demonstrated in Section 4 . Finally, a conclusion is drawn in Section 5.

\section{Local stability and Hopf bifurcation of the system (1.1)}

\subsection{Equilibria of the system (1.1)}

The equilibria of the system (1.1) are the solutions of the following system of algebraic equations

$$
a(y-x)=0, \quad-x z=0, \quad x y+e x^{2}-b=0,
$$

which reads

$$
S_{+}=\left(\sqrt{\frac{b}{e+1}}, \sqrt{\frac{b}{e+1}}, 0\right) \text { and } S_{-}=\left(-\sqrt{\frac{b}{e+1}},-\sqrt{\frac{b}{e+1}}, 0\right) .
$$

\subsection{Local stability of $S_{ \pm}$}

Noticing that the system (1.1) is invariant under the change of coordinates $(x, y, z) \rightarrow(-x,-y, z)$, i.e., symmetric with respective to the $\mathrm{z}$-axis, it follows that the stability of the equilibrium $S_{-}$can be obtained from the one of $S_{+}$. It suffices to investigate the behavior of $S_{+}$.

Lemma 2.2.1. The following statements are true.

(i) If $-1<e<-0.5$, then $S_{+}$is stable.

(ii) If $e>-0.5$, then $S_{+}$is unstable.

Proof. Set

$$
H=\left|\begin{array}{ccr}
a & 2 a b & 0 \\
1 & \frac{b}{e+1} & 0 \\
0 & a & 2 a b
\end{array}\right|
$$

Then all principal minors of $H$ are as follows.

$$
H_{1}=a, \quad H_{2}=\frac{-(2 e+1) a b}{e+1}, \quad H_{3}=2 a b H_{2} .
$$

By the Routh-Hurwitz criterion, the above consequence can be directly derived.

Lemma 2.2.1 immediately yields to the following conclusion.

Theorem 2.2.2. The following statements hold for the system (1.1).

(i) Suppose that $-1<e<-0.5$, then $S_{+}$and $S_{-}$are stable;

(ii) Suppose that $e>-0.5$, then $S_{+}$and $S_{-}$are unstable. 
HOPF BIFURCATION OF CODIMENSION ONE AND DYNAMICAL SIMULATION 463

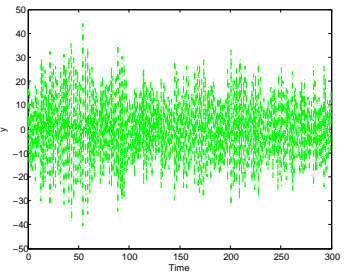

(a)

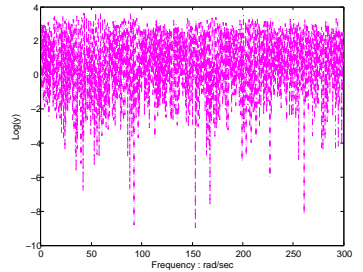

(b)

FIG. 2.1 (a) An apparent chaotic waveform of $y(t)$. (b) An apparent continuous broadband frequency spectrum $\log |y|$.

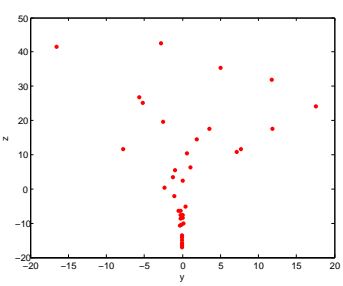

(a)

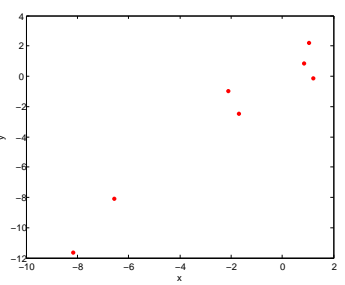

(c)

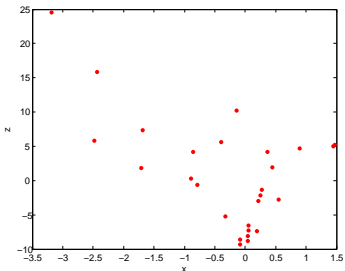

(b)

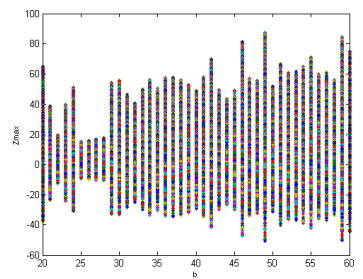

(d)

FIG. 2.2 Poincaré maps in the planes where (a) $x=0$, (b) $y=0$, (c) $z=0$, (d) A bifurcation diagram exhibiting a perioddoubling route to chaos of the peak of $z$ (' $z \max ^{\prime}$ ) of the system (1.1) versus the parameter $b$.

The above numerical observations show that small changes in the initial conditions of the system may cause big difference of its trajectories. For different initial conditions, the trajectories converge to different attractors. The above simulations also verify that, even though all of equilibria of the system (1.1) are stable, its trajectories can converge to two types of attractors (chaotic attractor or stable equilibria). The $z$-coordinate of the three attractors shows the sensitive dependence on its initial values (see Fig. 3.4(d)). 


\section{Forming mechanism of compound structure}

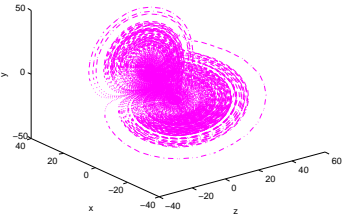

(a) Chaotic attractor of the system (1.1)

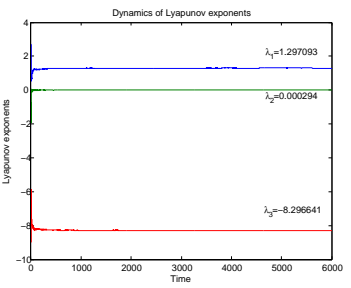

(b) Lyapunov exponents of the system (1.1)

FIG. 3.1 Chaotic attractor and Lyapunov exponents of the system $(1.1)$ with $(a, b, e)=(7,50,0.5)$ and the initial condition $\left(x_{0}, y_{0}, z_{0}\right)=(1,1,1)$.

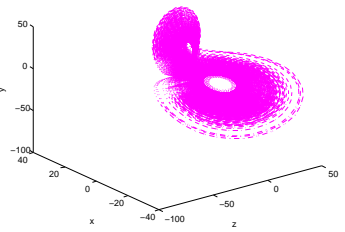

(a) Chaotic attractor of the system $(1.1)$

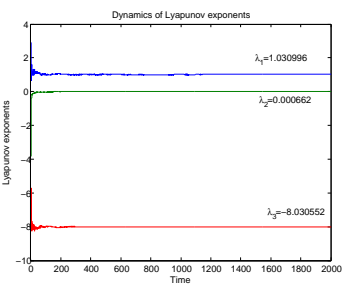

(b) Lyapunov exponents of the system (1.1)

FIG. 3.2 Chaotic attractor and Lyapunov exponents of the system $(1.1)$ with $(a, b, e)=(7,50,-0.5)$ and the initial condition $\left(x_{0}, y_{0}, z_{0}\right)=(1,1,1)$.

It is very interesting to consider the compound structure of a chaotic system [20]. Compound structures of the system (1.1) may be demonstrated using a half-image operation to obtain only the left or the right half-image attractors, both of which can then be merged together as a compound structure. Such an operation can be revealed through the use of a controlled system of the form:

$$
\left\{\begin{array}{l}
\dot{x}=a(y-x), \\
\dot{y}=-x z+u, \\
\dot{z}=-b+x y+e x^{2},
\end{array}\right.
$$

where $u$ is a control parameter, and $(a, b, e)=(7,50,0.1)$. 
HOPF BIFURCATION OF CODIMENSION ONE AND DYNAMICAL SIMULATION 465

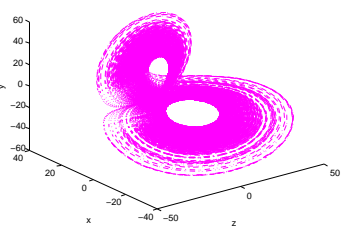

(a) Chaotic attractor of the system (1.1)

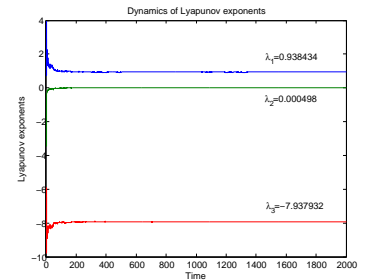

(b) Lyapunov exponents of the system $(1.1)$

Fig. 3.3 Chaotic attractor and Lyapunov exponents of the system $(1.1)$ with $(a, b, e)=(7,50,-0.54)$ and the initial condition $\left(x_{0}, y_{0}, z_{0}\right)=(1,1,1)$.

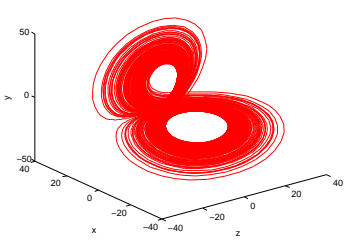

(a)

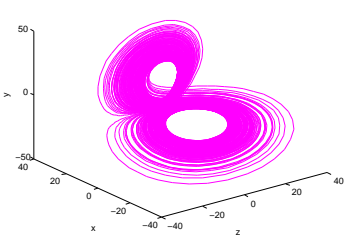

(c)

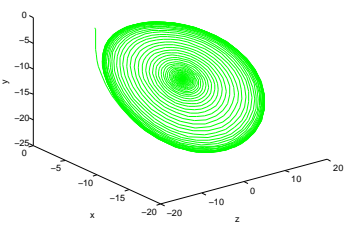

(b)

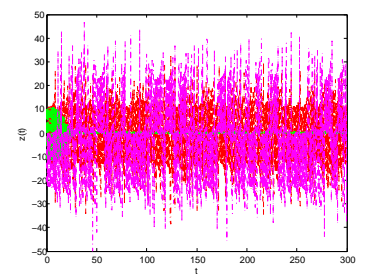

(d)

FIG. 3.4 Attractors of the system (1.1) with $a=7$, $b=50, e=-0.54$, and different initial condition $\left(x_{0}, y_{0}, z_{0}\right): \quad$ (a) $\left(x_{0}, y_{0}, z_{0}\right)=(-0.03,-5.1,-5.115) \quad$ (b) $\left(x_{0}, y_{0}, z_{0}\right)=(-0.03,-5.2,-5.1)$, and (c) $\left(x_{0}, y_{0}, z_{0}\right)=$ $(-0.03,-5.19,-5.15)$; (d) Time series of $z(t)$ with the above different initial values.

\subsection{Hopf bifurcation of $S_{ \pm}$}

It follows from Lemma 2.2.1 that there is an occurrence of bifurcation at $S_{+}$ for $e=-0.5$. Namely, $e=-0.5$ is a critical value, denoting $e_{0}=-0.5$. Then, what kind of bifurcation is it? Next, one will positively answer the question. 
For $e=e_{0}$, it is not difficult to deduce that Eq.(2.2) has a pair of conjugate purely imaginary roots $\lambda_{1,2}= \pm \sqrt{2 b} i$ and one negative real root $\lambda_{3}=-a$. So, $S_{+}$is then non-hyperbolic. From the Center Manifold Theorem, at the equilibrium point $S_{+}$, a $2 \mathrm{D}$ center manifold is well-defined and it is invariant under the flow generated by the system (1.1) (see [12] $P_{152}$ ). In view of Hopf bifurcation theory, one cane see that a Hopf bifurcation will occur at this time.

Lemma 3.1.1. The system (1.1) will undergo a Pioncaré-Andronov-Hopf bifurcation (or simply, Hopf bifurcation) at $S_{+}$when $e=e_{0}$.

Proof. Taking into account that $\operatorname{Re}\left(\lambda_{1}\right)=0$ at $e_{0}$, one obtains

$$
\left.\frac{d R e\left(\lambda_{1}\right)}{d e}\right|_{e=e_{0}}=\frac{2 a b}{a^{2}+2 b}>0 .
$$

So, the transversality condition holds, which, together with $\operatorname{Re}\left(\lambda_{3}\right)=-a \neq 0$, reads that all conditions for Hopf bifurcation to occur are satisfied. Consequently, the Hopf bifurcation happens at $S_{+}$when $e=e_{0}$.

In view of the Hopf theorem [12], this gives rise to the birth of a closed orbit near the equilibrium point $S_{+}$at $e=e_{0}$, which has initial period given by $T=\frac{2 \pi}{\omega}$, where $\omega=\operatorname{Im}\left(\lambda_{1}\right)=\sqrt{2 b}$. Then, how about the stability of the closed orbit? Now, one continues to study the direction of the Hopf bifurcation by utilizing the Project Method described in [12].

Consider the differential equation

$$
\dot{x}=A x+F(x, \alpha),
$$

where $x \in \mathbb{R}^{3}$ is a vector representing phase variables and $\alpha \in \mathbb{R}$ is a parameter variable. Assume that $F$ is of class $C^{\infty}$ in $\mathbb{R}^{3} \times \in \mathbb{R}$, whose components have Taylor expansion in $x$ starting with at least quadratic terms, $F=O\left(\|x\|^{2}\right)$.

Suppose $((0,0,0), 0)$ is an equilibrium point of $(2.3)$ where the Jacobian matrix $A$ has a pair of purely imaginary eigenvalues $\lambda_{2,3}= \pm i \omega_{0}, \omega_{0}>0$, and admits no other eigenvalue with zero real part. Let $T^{c}$ be the generalize eigenspace of $A$ corresponding to $\lambda_{2,3}$. By this it is meant the largest subspace invariant by $A$ on which the eigenvalues are $\lambda_{2,3}$.

Let $p, q \in \mathbb{C}^{3}$ be vectors such that

$$
A q=i \omega_{0} q, \quad A^{T} p=-i \omega_{0} p, \quad\langle p, q\rangle=\sum_{i=1}^{3} \bar{p}_{i} q_{i}=1
$$

where $A^{T}$ is the transposed of the matrix $A$. Any vector $x \in T^{c}$ can be parameterized as $x=z q+\bar{z} \bar{q}$, where $z=\langle p, x\rangle$. Then, Eq.(2.3) with $\alpha=0$ can be transformed into a single equation

$$
\dot{z}=i \omega_{0} z+g(z, \bar{z}, 0)
$$


where $g(z, \bar{z}, 0)=\langle p, F(z q+\bar{z} \bar{q}, 0)\rangle$ with a formal Taylor series expansion in two variable $z$ and $\bar{z}$

$$
g(z, \bar{z}, 0)=\sum_{k+l \geq 2} \frac{1}{k ! l !} g_{k l}(0) z^{k} \bar{z}^{l}
$$

where

$$
g_{k l}(0)=\frac{\partial^{k+l}}{\partial z^{k} \partial \bar{z}^{l}}\langle p, F(z q+\bar{z} \bar{q}, 0)\rangle
$$

for $k+l \geq 2, k, l=0,1, \ldots$.

Suppose that at $\alpha=0$ the function $F(x, \alpha)$ in $(2.3)$ is represented as

$$
F(x, 0)=\frac{1}{2} B(x, x)+\frac{1}{6} C(x, x, x)+O\left(\|x\|^{4}\right),
$$

where $B(x, y)$ and $C(x, y, z)$ are symmetric multi-linear vector functions of $x, y, z \in \mathbb{R}^{3}$. In coordinates, one has

$$
B_{i}(x, y)=\left.\sum_{j, k=1}^{3} \frac{\partial F_{i}(\xi, 0)}{\partial \xi_{j} \partial \xi_{k}}\right|_{\xi=0} x_{j} y_{k}, i=1,2
$$

and

$$
C_{i}(x, y, z)=\left.\sum_{j, k, l=1}^{3} \frac{\partial F_{i}(\xi, 0)}{\partial \xi_{j} \partial \xi_{k} \partial \xi_{l}}\right|_{\xi=0} x_{j} y_{k} z_{l}, i=1,2 .
$$

Then, some Taylor coefficients in $g(z, \bar{z}, 0)$ can be expressed by the formulas

$$
g_{20}=\langle p, B(q, q)\rangle, g_{11}=\langle p, B(q, \bar{q})\rangle, g_{02}=\langle p, B(\bar{q}, \bar{q})\rangle, g_{21}=\langle p, C(q, q, \bar{q})\rangle
$$

and the first Lyapunov coefficient is defined as

$$
l_{1}(0)=\frac{1}{2 \omega_{0}^{2}} \operatorname{Re}\left(i g_{20} g_{11}+\omega_{0} g_{21}\right)
$$

A Hopf bifurcation point $((0,0,0), 0)$ is an equilibrium point of $(2.3)$ where the Jacobian matrix A only has a pair of purely imaginary eigenvalues $\lambda_{2,3}=$ $\pm i \omega_{0}$ with $\omega_{0}>0$, and the other eigenvalue with non-zero real part. At a Hopf point a two dimensional center manifold is well defined, it is invariant under the flow generated by (2.3) and can be continued with arbitrary high class of differentiability to nearby parameter values.

A Hopf point is called transversal if the parameter-dependent complex eigenvalues cross the imaginary axis with nonzero derivative. In a neighborhood of a transversal Hopf point with $l_{1}(0)=0$ the dynamic behavior of the system (2.3), reduced to the family of parameter-dependent continuations of the center manifold, is orbitally topologically equivalent to the following complex normal form $\omega^{\prime}=(\eta+i) \omega+l_{1} \omega|\omega|^{2}$, where $\omega \in \mathbb{C}, \eta, l_{1}$ are real functions having derivatives of arbitrary higher order, which are continuations of $0, \omega_{0}$ and the first Lyapunov coefficient at the Hopf point [12]. As $l_{1}<0\left(l_{1}>0\right)$ one family of stable (unstable) periodic orbits can be found on this family of manifolds, shrinking to an equilibrium point at the Hopf point. 
One will employ the three-dimensional Hopf bifurcation theory and some computational results to perform the analysis of parametric variations with respect to dynamical bifurcations.

One is now in a position to evaluate the first Lyapunov coefficient of the system (1.1) at $S_{+}$. Note that, corresponding to the system (1.1), one takes $\alpha=e-e_{0}$, not $\alpha=(a, b, e)^{T}$, regarding $a, b$ as fixed in $(0, \infty)$.

Lemma 3.1.2. The first Lyapunov coefficient of the system (1.1) at $S_{+}$for $e=e_{0}$ is

$$
l_{1}(0)=\frac{a^{3} \sqrt{2 b}\left(5 a^{2}+16 b\right)}{64\left(a^{2}+2 b\right)^{3} b} .
$$

Proof. When $e=e_{0}, S_{+}=(\sqrt{2 b}, \sqrt{2 b}, 0)$. Take the variable change

$$
\left\{\begin{array}{l}
x_{1}=x-\sqrt{2 b} \\
x_{2}=y-\sqrt{2 b} \\
x_{3}=z
\end{array}\right.
$$

which transforms the equilibrium point $S_{+}$to the origin $\mathrm{O}(0,0,0)$ and the system (1.1) into

$$
\left\{\begin{array}{l}
\dot{x_{1}}=a\left(x_{2}-x_{1}\right) \\
\dot{x_{2}}=-x_{1} x_{3}-\sqrt{2 b} x_{3} \\
\dot{x_{3}}=\sqrt{2 b} x_{2}+x_{1} x_{2}-\frac{1}{2} x_{1}^{2} .
\end{array}\right.
$$

Using the notations in $(2.3)$, one has

$$
\begin{aligned}
& A=\left(\begin{array}{ccr}
-a & a & 0 \\
0 & 0 & -\sqrt{2 b} \\
0 & \sqrt{2 b} & 0
\end{array}\right), \\
& F(x)=\left(\begin{array}{c}
F_{1}(x) \\
F_{2}(x) \\
F_{3}(x)
\end{array}\right)=\left(\begin{array}{c}
0 \\
-x_{1} x_{3} \\
x_{1} x_{2}-\frac{1}{2} x_{1}^{2}
\end{array}\right) \text { for } x=\left(\begin{array}{l}
x_{1} \\
x_{2} \\
x_{3}
\end{array}\right) .
\end{aligned}
$$

It is easy to see that $A$ has a pair of conjugately purely imaginary eigenvalues $\lambda_{2,3}= \pm i \omega_{0}$ with $\omega_{0}=\sqrt{2 b}>0$ and a real eigenvalue $-a$. It is also easily obtained

$$
B(x, y)=\left(\begin{array}{c}
0 \\
-x_{1} y_{3}-x_{3} y_{1} \\
-x_{1} y_{1}+x_{1} y_{2}+x_{2} y_{1}
\end{array}\right) \text { for any } x=\left(\begin{array}{l}
x_{1} \\
x_{2} \\
x_{3}
\end{array}\right) \text { and } y=\left(\begin{array}{l}
y_{1} \\
y_{2} \\
y_{3}
\end{array}\right)
$$

and $C(x, x, x)=0$.

By some tedious calculations, one finds that

$$
p=\left(\begin{array}{c}
0 \\
i \\
1
\end{array}\right) \text { and } q=\frac{1}{2}\left(\begin{array}{c}
\frac{a(\sqrt{2 b}+a i)}{a^{2}+2 b} \\
i \\
1
\end{array}\right)
$$


HOPF BIFURCATION OF CODIMENSION ONE AND DYNAMICAL SIMULATION 469 satisfy

$$
A q=i \omega_{0} q, \quad A^{T} p=-i \omega_{0} p, \quad\langle p, q\rangle=\sum_{i=1}^{3} \bar{p}_{i} q_{i}=1 .
$$

Thereout, one has

$$
B(q, q)=\left(\begin{array}{c}
0 \\
-2 q_{1} q_{3} \\
-q_{1}^{2}+2 q_{1} q_{2}
\end{array}\right)=\left(\begin{array}{c}
0 \\
-\frac{a(\sqrt{2 b}+a i)}{2\left(a^{2}+2 b\right)} \\
\frac{a\left[-a\left(a^{2}+6 b\right)+4 b \sqrt{2 b} i\right]}{4\left(a^{2}+2 b\right)^{2}}
\end{array}\right)
$$

and

$$
B(q, \bar{q})=\left(\begin{array}{c}
0 \\
-q_{1} \overline{q_{3}}-q_{3} \overline{q_{1}} \\
-q_{1} \overline{q_{1}}+q_{1} \overline{q_{2}}+q_{2} \overline{q_{1}}
\end{array}\right)=\left(\begin{array}{c}
0 \\
-\frac{a \sqrt{2 b}}{2\left(a^{2}+2 b\right)} \\
\overline{4\left(a^{2}+2 b\right)}
\end{array}\right) .
$$

Therefore, further computations lead to

$$
\begin{aligned}
g_{20} & =\frac{a(-a+\sqrt{2 b} i)}{2\left(a^{2}+2 b\right)}+\frac{a\left[-a\left(a^{2}+6 b\right)+4 b \sqrt{2 b} i\right]}{4\left(a^{2}+2 b\right)^{2}} \\
& =\frac{a\left[-a\left(3 a^{2}+10 b\right)+2 \sqrt{2 b}\left(a^{2}+4 b\right) i\right]}{4\left(a^{2}+2 b\right)^{2}}, \\
g_{11} & =\frac{a(a+2 \sqrt{2 b} i)}{4\left(a^{2}+2 b\right)}, \quad \text { and } \quad g_{21}=0 .
\end{aligned}
$$

So, the first Lyapunov coefficient for Eq.(1.1) takes the value

$$
\begin{aligned}
l_{1}(0) & =\frac{1}{2 \omega_{0}^{2}} \operatorname{Re}\left(i g_{20} g_{11}+\omega_{0} g_{21}\right) \\
& =\frac{1}{4 b} \times \frac{-a^{2}\left[-a\left(3 a^{2}+10 b\right) 2 \sqrt{2 b}+2 a \sqrt{2 b}\left(a^{2}+4 b\right)\right]}{16\left(a^{2}+2 b\right)^{3}} \\
& =\frac{a^{3} \sqrt{2 b}\left(5 a^{2}+16 b\right)}{64\left(a^{2}+2 b\right)^{3} b} .
\end{aligned}
$$

Obviously, $l_{1}(0)>0$ for $a, b \in(0, \infty)$, hence, the closed orbit should be unstable. Combining Lemma 2.3.1 and Lemma 2.3.2, one obtains the following Hopf bifurcation consequences.

Theorem 3.1.3. The system (1.1) respectively undergoes a Hopf bifurcation at $S_{+}$and $S_{-}$for $e=e_{0}$ with the first Lyapunov coefficient

$$
l_{1}(0)=\frac{a^{3} \sqrt{2 b}\left(5 a^{2}+16 b\right)}{64\left(a^{2}+2 b\right)^{3} b}>0 .
$$

So, the system (1.1) has two transversal Hopf points: $S_{+}$and $S_{-}$, which are unstable (weak repelling center for the flow of the system (1.1) restricted to the center manifold). Moreover, for each $e<e_{0}$ but close to $e_{0}$, there exists an unstable limit cycle near $S_{+}$and $S_{-}$, respectively. 


\section{Some other properties of the system (1.1)}

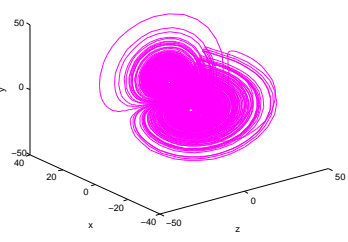

(a)

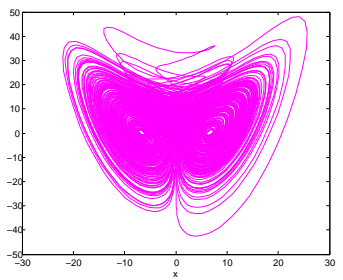

(c)

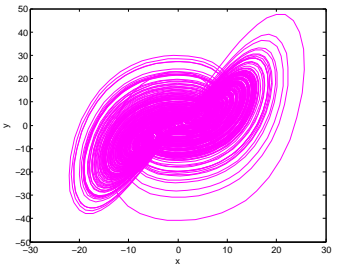

(b)

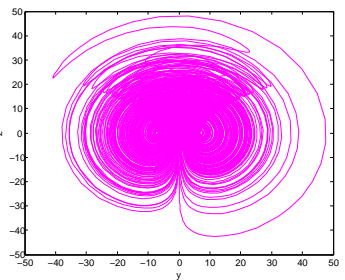

(d)

FiG. 4.1 Phase portraits of the system (1.1) with $a=7$, $b=50, e=0.1$, the initial condition $\left(x_{0}, y_{0}, z_{0}\right)=(1,1,1):$ (a) in the space $\mathbb{R}^{3}$, and its projections (b) in the plane $\mathrm{x}-\mathrm{y},(\mathrm{c})$ in the plane $\mathrm{x}-\mathrm{z},(\mathrm{d})$ in the plane $\mathrm{y}-\mathrm{z}$.

\subsection{Lyapunov exponent}

It is known that Lyapunov exponent is the exponential rate of divergence or convergence near trajectories in the phase space. Anyone system containing at least one positive Lyapunov exponent is to be defined to be chaotic [34]. When $(a, b, e)=(7,50,1)$ and the initial values $\left(x_{0}, y_{0}, z_{0}\right)=(1,1,1)$, the Lyapunov exponents of the system (1.1) are found to be $L_{1}=1.139490, L_{2}=0.000057$ and $L_{3}=-8.138626$ as mentioned above. In addition, the Lyapunov dimension of the system (1.1) is fractional as described by

$$
D_{L}=j+\frac{1}{\left|L_{j+1}\right|} \sum_{i=1}^{j} L_{i}=2.1400 .
$$

\subsection{Dissipativity}

The divergence of flow of the dynamic system (1.1) is

$$
\nabla V=\frac{\partial \dot{x}}{\partial x}+\frac{\partial \dot{y}}{\partial y}+\frac{\partial \dot{z}}{\partial z}=-a
$$


HOPF BIFURCATION OF CODIMENSION ONE AND DYNAMICAL SIMULATION 471

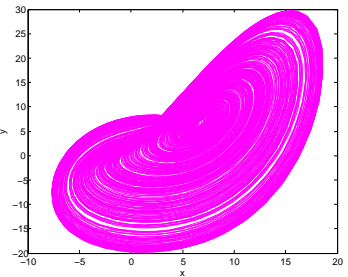

(a)

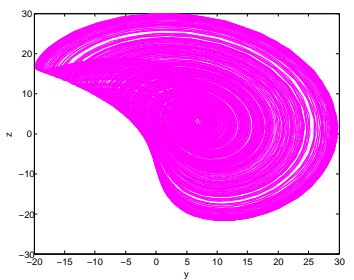

(c)

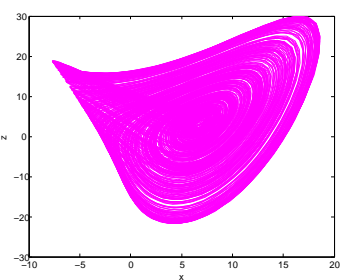

(e)

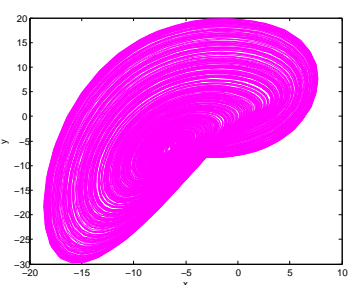

(b)

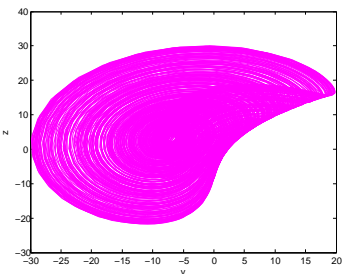

(d)

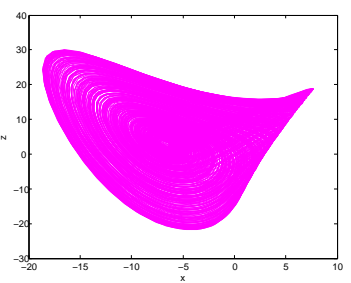

(f)

Fig. 4.2 (a) A right half-image attractor for the $\mathrm{x}-\mathrm{y}$ plane at $u=20$. (b) A left half-image attractor for the $\mathrm{x}-\mathrm{y}$ plane at $u=-20$. (c)A right half-image attractor for the $\mathrm{y}-\mathrm{z}$ plane at $u=20$. (d) A left half-image attractor for the $\mathrm{y}-\mathrm{z}$ plane at $u=-20$. (e) A right half-image attractor for the $\mathrm{x}-\mathrm{z}$ plane at $u=20$. (f) A left half-image attractor for the $\mathrm{x}-\mathrm{z}$ plane at $u=-20$.

As $a>0$, the system (1.1) is a dissipative one with an exponential rate of contraction as

$$
\frac{d V}{d t}=\exp (-a)
$$

In other words, a volume element $V_{0}$ becomes smaller by the flow into a volume element $V_{0} \exp (-a t)$ in time $t$. Each volume containing the trajectories of this system shrinks to zero as $t \rightarrow \infty$ at an exponential rate $-a$. The orbits in this 


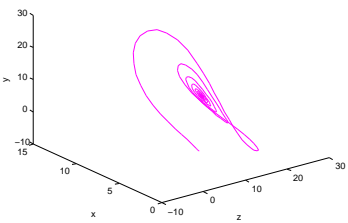

(a)

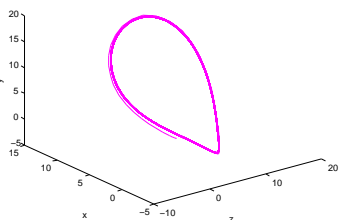

(c)

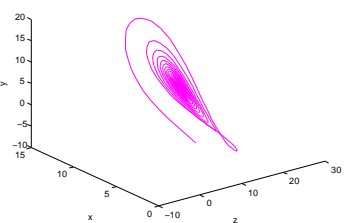

(b)

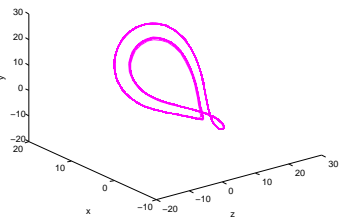

(d)

FIG. 4.3 Phase portraits of the system (4.1) at (a) $u=90$; (b) $u=60$; (c) $u=40$; (d) $u=28$;

system are ultimately confined into a specific motion settling onto an attractor of the system [17].

\subsection{Time domain, frequency spectrum, Poincaré map and bifurca- tion}

When $(a, b, e)=(7,50,1)$ and the initial value vector $\left(x_{0}, y_{0}, z_{0}\right)=(1,1,1)$, Fig. 2.1(a) shows an apparent chaotic waveform $\mathrm{y}(\mathrm{t})$ of the system (1.1) whilst, in the frequency domain, an apparent continuous broadband spectrum $\log |y|$ of the system (1.1) is shown in Fig. 2.1(b) in the time domain. It can be seen from Fig. 2 that the new system exhibits chaotic behavior. Figs. 2.2(a), (b) and (c) visualize the Poincaré maps in the planes where $x=0, y=0$ and $z=0$, respectively. Several sheets of the attractors are displayed. It is noticeable that the Poincaré maps of many chaotic systems such as the generalized Lorenz system (GLS) [3] only show a branch with several twigs. The Poincaré maps in Figs. 2.2(a) and (b), however, consist of virtually asymmetrical branches and a number of nearly asymmetrical twigs. In Fig. 2.2(c), it is clear that few sheets are folded. As an example, Fig. 2.2(d) shows a bifurcation diagram exhibiting a period-doubling route to chaos of the peak of $z$ (' $z \max$ ') of the system (1.1) versus the parameter $b$ which is varied from 20 to 60 . There are some periodic windows in the chaotic region. The chaotic behavior disappears quickly if $b<0$. It is apparent from Fig. 4 that ' $z$ max' is the highest near $b=50$. Similarly, ' $x$ max' and ' $y$ max' in bifurcation diagrams are also the highest near $b=50$. As a result, the value of $b$ described in the system (1.1) has been chosen to be 50 . 


\subsection{Chaotic attractor coexistence with three types of equilibria}

By using several traditional ways of describing chaotic attractor, we will show that this system is indeed chaotic. (Note that the system (1.1) has a chaotic attractor if $L_{1}>0, L_{2}=0$ and $L_{3}<0$.) Moreover, numerical simulations illustrate that this system possesses complicated and unusual dynamical behaviors despite of its simple form.

When the parameters $a=7, b=50$ and $e$ varies, we analyze the evolution process of this system and dynamical behavior with the initial values $(1,1,1)$.

For the parameter $e=0.5$, a double-scroll chaotic attractor is displayed with the Lorenz-like shape in Fig. 3.1(a) together with the corresponding exponents in Fig. 3.1(b). At this time, the eigenvalues $\lambda_{2,3}=1.6658 \pm 8.0609 i$ indicate that the equilibria $S_{ \pm}$are both unstable.

For the parameter $e=-0.5$, one observes that the system (1.1) has a chaotic attractor (see Fig. 3.2), with the characteristic values $\lambda_{1}=-7$ and $\lambda_{2,3}= \pm 10 i$, which says two non-hyperbolic equilibria. Therefore, the equilibria $S_{+}$ and $S_{-}$respectively have a local $1 \mathrm{D}$ stable manifold tangent to the eigenvector associated to the eigenvalue $\lambda_{1}$ and a local $2 \mathrm{D}$ center manifold tangent to the plane generated by the eigenvectors associated to two conjugate complex eigenvalues $\lambda_{2,3}$.

For the parameter $e=-0.54$, another type of chaotic attractor can be observed (see Fig. 3.3(a)) in this system. Its Lyapunov exponents also show that this system is chaotic. It is noted that, the chaotic attractor is nearly the same as the chaotic attractor plotted in Figs. 3.1 and 3.2, although both equilibria $S_{ \pm}$are stable (because its characteristic values are $\lambda_{1}=-6.602$, $\left.\lambda_{2,3}=-0.1999 \pm 10.2965 i\right)$. Therefore, the system (1.1) has neither homoclinic orbits nor heteroclinic orbits joining $S_{+}$and $S_{-}$.

To clearly show the three types of chaotic attractors mentioned previously, we list the equilibria, the eigenvalues, the Lyapunov exponents and the fractional dimensions for typical parameter values in Table 1.

Now, consider the influence of the initial conditions on the dynamics of this system with the fixed parameters values. In particular, when we fix $(a, b, e)=$ $(7,50,-0.54)$ and change the initial values slightly, dynamical behaviors of the system (1.1) may produce large variations in the long term. Besides the two stable equilibrium points, chaotic attractors of the system (1.1) also are obtained, which imply that chaos coexists with the two stable fixed points:

(i) A chaotic attractor with the initial values $(-0.03,-5.1,-5.115)$ is shown as Fig. 3.4(a). The Lyapunov exponents of the system are found to be $L_{1}=$ $0.902350, L_{2}=-0.000178$ and $L_{3}=-7.901210$;

(ii) For the initial values $(-0.03,-5.2,-5.1)$, its trajectories converge to the stable equilibrium $S_{+}$(see Fig. 3.4(b)) and the Lyapunov exponents of the system are found to be $L_{1}=-0.043117, L_{2}=-0.171874$ and $L_{3}=-6.784995$; 
TABLE 1 Numerical analysis for several typical chaotic attractors of the system (1) with the parameter vector $(a, b)=$ $(7,50)$ and varying $e$ at the initial values $(1,1,1)$.

\begin{tabular}{lllll}
\hline Parameter $e$ & Equilibria & Eigenvalues & LEs & Fractional dimension \\
\hline & & & & \\
$e=0.5$ & $( \pm 5.7735, \pm 5.7735,0)$ & $\lambda_{1}=-10.3316$ & $L_{1}=1.297093$ & \\
& & $L_{2}=0.000294$ & $D_{L}=2.1546$ \\
& & & $L_{3}=-8.296641$ & \\
$e=-0.5$ & $( \pm 10, \pm 10,0)$ & $\lambda_{1}=-7$ & $L_{1}=1.030996$ & \\
& & $\lambda_{2,3}= \pm 10 i$ & $L_{2}=0.000662$ & $D_{L}=2.1258$ \\
& & & $L_{3}=-8.030552$ & \\
$e=-0.54$ & $( \pm 10.4257, \pm 10.4257,0)$ & $\lambda_{1}=-6.6002$ & $L_{1}=0.938434$ & \\
& & $\lambda_{2,3}=-0.1999 \pm 10.6925 i$ & $L_{3}=-7.937932$ & \\
& & &
\end{tabular}

(iii) A chaotic attractor with the initial values $(-0.03,-5.19,-5.15)$ is shown as Fig. 3.4(c) with the Lyapunov exponents $L_{1}=-0.918365, L_{2}=0.000779$ and $L_{3}=-7.918202$.

When $u=20$, a left half-image of the original attractor (Fig. 4.1) can be isolated as illustrated in, for example, Fig. 4.2(a) for the x-y plane, Fig. 4.2(c) for the $\mathrm{y}-\mathrm{z}$ plane and Fig. 4.2(e) for the $\mathrm{x}-\mathrm{z}$ plane, respectively.

In contrast, when $u=-20$, another right half-image of the original attractor (Fig. 4.1) can be isolated as illustrated in Fig. 4.2(b) for the x-y plane, Fig. 4.2(d) for the $\mathrm{y}-\mathrm{z}$ plane and Fig. 4.2(f) for the $\mathrm{x}-\mathrm{z}$ plane, respectively.

In other words, Fig. 4.1(b) is separated into Figs. 4.2(a) and 4.2(b), Fig. 4.1(c) is separated into Figs. 4.2(e) and 4.2(f), and Fig. 4.1(d) is separated into Figs. 4.2(c) and 4.2(d). It can be seen from Fig. 4.2 that the new system consists of compound structures. Figs. 3.1-3.4 demonstrate gradual development of forming mechanisms of such compound structures.

With the help of computer observations, its gradual development processes are influenced by the parameter $u$ as much as the initial values, which verify the complex dynamics behavior of chaotic system for a second time. In spite of those, we get the following statements by simulations under setting the initial value $(1,1,1)$.

Different dynamical behaviors can be summarized as follows:

(i) When $|u| \geq 75$, the system (4.1) converges to a point (see Fig. 4.3(a)).

(ii) When $53 \leq|u|<75$, the system (4.1) is the transition between the limit cycle and limit point (see Fig. 4.3(b)).

(iii) When $29 \leq|u| \leq 53$, the system (12) has limit cycles. Fig. 4.3(c) shows a limit cycle at $\mathrm{u}=40$.

(iv) When $26.2 \leq|u| \leq 29$, the system (4.1) demonstrates period doubling bifurcation. For example, see Fig. 4.3(d).

(v) When $25.7<|u|<26.2$, the system (4.1) demonstrates period three bifurcations. Refer to Fig. 4.4(a). 


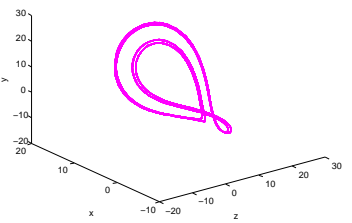

(a)

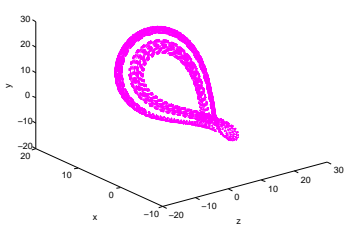

(c)

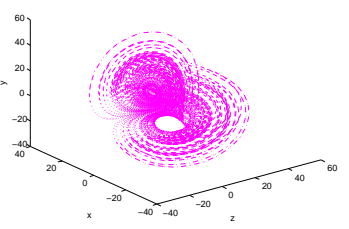

(e)

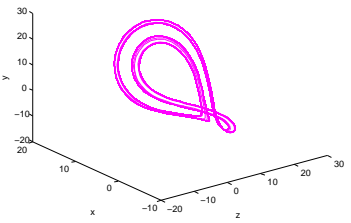

(b)

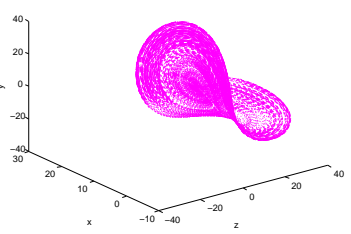

(d)

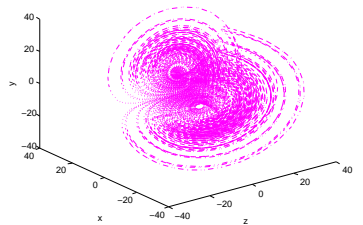

(f)

FIG. 4.4 Phase portraits of the system (4.1) at (a) $u=26$; (b) $u=25.5$; (c) $u=25$; (d) $u=19$; (e) $u=10$; (f) $u=1$.

(vi) When $25.3<|u| \leq 25.7$, the system (4.1) demonstrates period four bifurcations (see Fig. 4.4(b)).

(vii) When $24<|u| \leq 25.3$, the system (4.1) demonstrates the transition between the half imagine and period four bifurcations. Refer to Fig. 4.4(c).

(viii) When $18.3 \leq|u| \leq 24$, the system (4.1) becomes a left (or a right) half-image attractor [e.g. see Fig. 3.2(a)-(f)]. Refer to Fig. 4.4(d).

(ix) When $5 \leq|u| \leq 18.2$, the system demonstrates partial attractors, which are bounded. Fig. 4.4(e) shows a partially-left, dominantly-right, attractor at $u=10$.

(x) When $|u|<5$, the system exhibits a complete attractor. For example, Fig. 4.4(f) shows the complete attractor at $u=1$.

\section{Conclusion}

In this paper, a chaotic system is formulated with three nonlinearity in the form of quadratic terms. The new chaotic attractors coexisting with stable or 
unstable or non-hyperbolic equilibria are different from the other attractor in the Lorenz and Lorenz-type systems except the system [35].

What we most want to formulate is the fact that, the system (1.1) with only stable or non-hyperbolic equilibria, simpler than the system [35], still may generate chaos.

Theoretically, mainly, its Hopf bifurcation of codimension one is in detail considered.

Although the form of the system (1.1) looks simple, it has compound dynamical behaviors, which, by simulations, may be demonstrated in the terms of the Lyapunov exponent, the dissipativity with the existence of the attractor, the apparent chaotic waveform in time domain, the apparent chaotic continuous broadband frequency spectrum, the Poincaré map, and the bifurcation, etc.

Maybe these simulations are not too difficult, and similar work can also be found in $[6,17,20]$, etc. However, what we would like to state here is to show the forming mechanism of compound chaos structure by a detailed case. The strict theoretical verifications for the results simulated are still open by now. These simulation results also shows that the system (1.1) possesses abundant and complex dynamical properties not solved theoretically, which desire to be further investigated.

The fact, we state here, that a simpler system with only stable or nonhyperbolic equilibria still may generate chaos maybe provides some new insights into the forming mechanics of chaos, and is hoped to shed some light to final revealing the true geometrical structure of the amazing original Lorenz attractor, which is our willing to present this paper.

\section{References}

[1] G. Alvarez, S. Li, F. Montoya, G. Pastor, and M. Romera, Breaking projective chaos sychronization secure communication using filtering and generalized synchronization, Chaos Solitons Fractals 24 (2005), no. 3, 775-783.

[2] H. Asakura, K. Takemura, Z. Yoshida, and T. Uchida, Collisionless heating of electrons by meandering chaos and its application to a low-pressure Plasma source, Jpn. J. Appl. Phys. 36 (1997), 4493-4496.

[3] S. Čelikovský and G. Chen, On a generalized Lorenz canonical form of chaotic systems, Internat. J. Bifur. Chaos Appl. Sci. Engrg. 12 (2002), no. 8, 1789-1812.

[4] _ Hyperbolic-type generalized Lorenz system and its canonical form, Proc. 15th Triennial World Congrss of IFAC, Barcelona, Spain, (2002b), in CD ROM.

[5] _ On the generalized Lorenz canonical form, Chaos Solitons Fractals 26 (2005), no. 5, 1271-1276.

[6] S. Čelikovský and A. Vaěček, Bilinear systems and chaos, Kybernetika 30 (1994), no. 4, 403-424.

[7] _ Control Systems: from linear analysis to synthesis of chaos, London, PrenticeHall, 1996.

[8] G. Chen and T. Ueta, Yet another chaotic attractor, Internat. J. Bifur. Chaos Appl. Sci. Engrg. 9 (1999), no. 7, 1465-1466.

[9] _ Bifurcation analysis of Chen's equation, Internat. J. Bifur. Chaos Appl. Sci. Engrg. 10 (2000), no. 8, 1917-1931. 
[10] L. O. Chua, M. Itoh, L. Kovurev, and K. Eckert, Chaos synchronization in Chua's circuit, J. Circuits Systems Comput. 3 (1993), no. 1, 93-108.

[11] K. Huang and G. Yang, Stability and Hopf bifurcation analysis of a new system, Chaos Solitons Fractals 39 (2009), no. 2, 567-578.

[12] Y. A. Kuznetsov, Elements of Applied Bifurcation Theory, second edition, SpringerVerlag, New York, 1998.

[13] C. Li and G. Chen, A note on Hopf bifurcation in Chen's system, Internat. J. Bifur. Chaos Appl. Sci. Engrg. 13 (2003), no. 6, 1609-1615.

[14] T. Li, G. Chen, and Y. Tang, Complex dynamical behaviors of the chaotic Chen's system, Internat. J. Bifur. Chaos Appl. Sci. Engrg. 13 (2003), no. 9, 2561-2574.

[15] - On stability and bifurcation of Chen's system, Chaos Solitons Fractals 19 (2004), no. 5, 1269-1282.

[16] X. Li and Q. Ou, Dynamical properties and simulation of a new Lorenz-like chaotic system, Nonlinear Dynam. 65 (2011), no. 3, 255-270.

[17] C. Liu, T. Liu, L. Liu, and K. Liu, A new chaotic attractor, Chaos Solitons Fractals 22 (2004), no. 5 1031-1038.

[18] E. N. Lorenz, Deterministic non-periodic flow, J. Atmospheric Sci. 20 (1963), 130-141.

[19] J. Lü and G. Chen, A new chaotic attractor coined, Internat. J. Bifur. Chaos Appl. Sci. Engrg. 12 (2002), no. 3, 659-661.

[20] J. Lü, G. Chen, and S. Zhang, The compound structure of a new chaotic attractor, Chaos Solitons Fractals 14 (2002), no. 5, 669-672.

[21] J. Lü, T. Zhou, G. Chen, and S. Zhang, Local bifurcations of the Chen system, Internat. J. Bifur. Chaos Appl. Sci. Engrg. 12 (2002), no. 10, 2257-2270.

[22] B. Munmuangsaen and B. Srisuchinwong, A new five-term simple chaotic attractor, Phys. Lett. A 373 (2009), 4038-4043.

[23] J. M. Ottino, C. W.Leong, H. Rising, and P. D. Swanson, Morphological structures produced by mixing in chaotic flows, Nature 333 (1988), 419-425.

[24] O. E. Rössler, An equation for continuous chaos, Phys. Lett. A 57 (1976), 397-398.

[25] C. P. Silva, S̆ilnikov theorem-a tutorial, IEEE Trans. Circuits Systems I Fund. Theory Appl. 40 (1993), no. 10, 657-682.

[26] L. P. S̆ ilnikov, A case of the existence of a countable number of periodic motions, Sov. Math. Docklady 6 (1965), 163-166.

[27] _ A contribution of the problem of the structure of an extended neighborhood of rough equilibrium state of saddle-focus type, Math. USSR-Shornik 10 (1970), 91-102.

[28] C. Sparrow, The Lorenz Equations: Bifurcations, Chaos, and Strange Attractors, Applied Mathematical Sciences, 41. Springer-Verlag, New York-Berlin, 1982.

[29] J. C. Sprott, Some simple chaotic flows, Phys. Rev. E (3) 50 (1994), no. 2, R647-R650.

[30] Simplest dissipative chaotic flow, Phys. Lett. A 228 (1997), no. 4-5, 271-274.

[31] — A new class of chaotic circuit, Phys. Lett. A 266 (2000), 19-23.

[32] G. Tigan and D. Constantinescu, Heteroclinic orbits in the T and the Lü system, Chaos Solitons Fractals 42 (2009), no. 1, 20-23.

[33] G. van der Schrier and L. Maas, The diffusionless Lorenz equations; Shilnikov bifurcations and reduction to an explicit map, Phys. D 141 (2000), no. 1-2, 19-36.

[34] A. Wolf, J. B. Swift, H. L. Swinney, and J. A. Vastano, Determining Lyapunov exponents from a time series, Phys. D 16 (1985), no. 3, 285-317.

[35] Z. Wei and Q. Yang, Dynamics of a new autonomous 3-D chaotic system only with stable equilibria, Nonl. Anal.: Real World Applications 12 (2011), 106-118.

[36] Q. Yang and G. Chen, A chaotic system with one saddle and two stable node-foci, Internat. J. Bifur. Chaos Appl. Sci. Engrg. 18 (2008), no. 5, 1393-1414.

[37] Q. Yang, G. Chen, and Y. Zhou, A unified Lorenz-type system and its canonical form, Internat. J. Bifur. Chaos Appl. Sci. Engrg. 16 (2006), no. 10, 2855-1871. 
[38] Y. Yu and S. Zhang, Hopf bifurcation in the Lü system, Chaos Solitons Fractals 17 (2003), no. 5, 901-906.

[39] Hopf bifurcation analysis of the Lü system, Chaos Solitons Fractals 21 (2004), no. $5,1215-1220$.

\section{XIANYI Li}

College of Mathematical Science

YANGZHOU UNIVERSITY

Yangzhou, Jiangsu 225002, P. R. China

E-mail address: mathxyli@yzu.edu.cn

ZhengXin Zhou

College of Mathematical Science

YANGZHOU UNIVERSITY

YAngzhou, Jiangsu 225002, P. R. China

E-mail address: zhengxinzhou@hotmail.com 